\title{
Discrete breathers in thermal equilibrium: distributions and energy gaps
}

\author{
M. Eleftheriou ${ }^{1,2}$ and S. Flach ${ }^{3}$ \\ ${ }^{1}$ Department of Physics, University of Crete P. O. Box 2208, \\ 71003 Heraklion, Crete, Greece \\ ${ }^{2}$ I. N. F. M. Unita di Firenze, Via G. Sansone 1, 50019 Sesto F.no, Italy \\ 3 Max Planck Institute for the Physics of Complex Systems, \\ Nöthnitzer Str. 38, D-01187 Dresden, Germany
}

December 22, 2018

\begin{abstract}
We study a discrete two-dimensional nonlinear system that allows for discrete breather solutions. We perform a spectral analysis of the lattice dynamics at thermal equilibrium and use a cooling technique to measure the amount of breathers at thermal equilibrium. Our results confirm the existence of an energy threshold for discrete breathers. The cooling method provides with a novel computational technique of measuring and analyzing discrete breather distribution properties in thermal equilibrium.
\end{abstract}

\section{Introduction}

Nonlinear discrete systems support discrete breathers (DBs). These timeperiodic and spatially localized solutions are the result of the interplay between nonlinearity and discreteness [1. Many studies of DBs have been successfully launched, on such topics as rigorous existence proofs, dynamical and structural stability and computational methods of obtaining DBs in classical models as well as their quantum aspects. In addition DBs have been detected and studied experimentally in such different systems as interacting Josephson junction systems [2], coupled nonlinear optical waveguides [3], lattice vibrations in crystals [4, antiferromagnetic structures [5], micromechanical cantilever arrays [6], Bose-Einstein condensates loaded on optical lattices [7, layered high- $T_{c}$ superconductors [8]. Their existence is also predicted to exist in the dynamics of dusty plasma crystals [9].

Among several intriguing unresolved questions concerning DBs, a central issue is the contribution of DBs to the dynamics of systems at thermal 
equilibrium. Indeed breather-like excitations have been observed in a variety of different models at finite temperatures [10. For some special models with additional conservation laws semianalytical statements about the contribution of DBs to thermal equilibrium have been derived [11. The question is then whether we can identify the contribution of DBs to various equilibrium and relaxation properties (like e.g. the charge trapping in DNA [12]). Since DBs are dynamical excitations, their contribution will be observable mainly in time-dependent (or frequency-dependent) correlation functions, while static correlation functions, e.g. specific heat, only probe the available energy landscape, and are not suitable for detecting dynamical correlations. A good way to proceed is to use a specific property of DBs and trace its contribution to correlation functions. Another question is whether we can design computational methods to separate the breather excitations at a given time from the rest of the excitations in the lattice. This would allow us to perform systematic studies of distribution properties of DBs in a given lattice at a given temperature.

Concerning the first point from above, we know that in general nonlinear systems in two and three dimensions support breather solutions that have a positive lower energy threshold [13. This is a very important property that can be of help in the detection of DBs in experiments. In fact for specific one-dimensional lattices this property holds as well. However the search for traces of these thresholds in correlation functions for one-dimensional lattices at thermal equilibrium turned out to be very complicated [14]. There are two reasons for that. First, DBs in one-dimensional lattices act as strong scatterers of plane waves [15. Consequently radiation can be efficiently trapped between DBs, and contribute to a strong interaction between DBs. It is also hard to find a way for letting the radiation out of the system (see [16]). Secondly frequency-dependent correlation functions probe gaps in frequency space. Although the existence of energy thresholds for breathers leads also to frequency thresholds, the values of these frequency thresholds may become too small to be easily detected [14.

In this work we examine a two-dimensional lattice. First fingerprints of the DB energy threshold in two-dimensional lattices have been reported in 17. and also in [18. A comprehensive study of DBs in thermal equilibrium and their influence on various relaxation mechanisms was provided by Ivanchenko et al [19]. We attempt to go sufficiently beyond these studies. Here we present not only the frequency dependence of correlation functions, but also their temperature (or energy) dependence. We then proceed to apply a cooling technique at the boundaries of our system to efficiently get rid of extended excitations in the lattice. The remaining localized breather-like excitations can then be easily analyzed. We observe the existence of energy thresholds and provide with novel distribution functions for DB energies. 


\section{Gap determination in the Hamiltonian system}

We study a two-dimensional quadratic lattice with one degree of freedom per site. The equation of motion for the particle at site $(i, j)$ in a lattice of size $N \times N$ with free ends is given by

$$
\ddot{u}_{i, j}=k\left(u_{i+1, j}+u_{i-1, j}-2 u_{i, j}\right)+k\left(u_{i, j+1}+u_{i, j-1}-2 u_{i, j}\right)-u_{i, j}-u_{i, j}^{3},
$$

where $u_{i, j}$ is the displacement of the particle at site $i, j$. The masses of the particles are equal to unity and the interaction between them is harmonic with strength $k$ while the on-site potential is of hard- $\phi^{4}$ type. The corresponding Hamiltonian from which (10) is derived, is given by

$$
H=\sum_{i, j} h_{i, j}, h_{i, j}=\frac{1}{2} \dot{u}_{i, j}^{2}+\frac{1}{2} u_{i, j}^{2}+\frac{1}{4} u_{i, j}^{4}+\frac{k}{4} \sum_{N N}\left(u_{i, j}-u_{l, m}\right)^{2} .
$$

Here $h_{i, j}$ is the discrete on-site energy density which will be used later, and $N N$ stands for nearest neighbors and implies $(l-i)^{2}+(m-j)^{2}=1$. For small amplitudes the linearized equation (11) yields plane waves $u_{l, m}(t) \sim$ $\mathrm{e}^{i\left(\omega_{q} t-q_{x} l-q_{y} m\right)}$ with the phonon dispersion relation

$$
\omega_{\vec{q}}^{2}=1+4 k\left(\sin ^{2}\left(\frac{q_{x}}{2}\right)+\sin ^{2}\left(\frac{q_{y}}{2}\right)\right)
$$

where $\vec{q} \equiv\left(q_{x}, q_{y}\right)$ is the wave vector of the reciprocal lattice.

We obtain the energy threshold for breathers using the Newton algorithm and the anticontinuous limit [1]. We compute breather solutions for different frequencies for coupling $k=0.05$. The phonon band of small amplitude plane waves is located between the frequencies $\omega_{\vec{q}=(0,0)} \equiv \omega_{0}=1$ and $\omega_{\vec{q}=(\pi, \pi)} \equiv \omega_{\pi}=1.183$. We use a lattice of $19 \times 19$ sites. In Fig. 1 we plot the total energy of the breather solution as a function of its frequency $\Omega_{b}$. Additionally we check the stability of the obtained solutions using the standard Floquet analysis 11. We observe in Fig. 1] that the curve of the total energy of the breather as a function of frequency consists of two branches, separated by a minimum at frequency $\Omega_{b, t h}$. In the left branch (dashed line) DBs are unstable according to the Floquet analysis (see Fig 2A) while in the right branch (solid line) DBs are linearly stable solutions (Fig $2 \mathrm{C})$. The breather with frequency $\Omega_{b, t h}=1.207$ is separating the frequency region $\omega_{\pi}<\Omega_{b}<\Omega_{b, t h}$ where breathers are unstable and the region $\Omega_{b, t h}<\Omega_{b}$ where breathers are linearly stable according to our computations. This specific breather solution has a total energy $E_{b, t h}=0.268$. $60 \%$ of the energy of this DB is located on the central site, i.e. $h_{\text {central }}=0.167$. In other words, the minimum energy breather is still a rather discrete object, involving essentially only a few lattice sites in its dynamics (Fig,2B) (see also [13, 20]). 


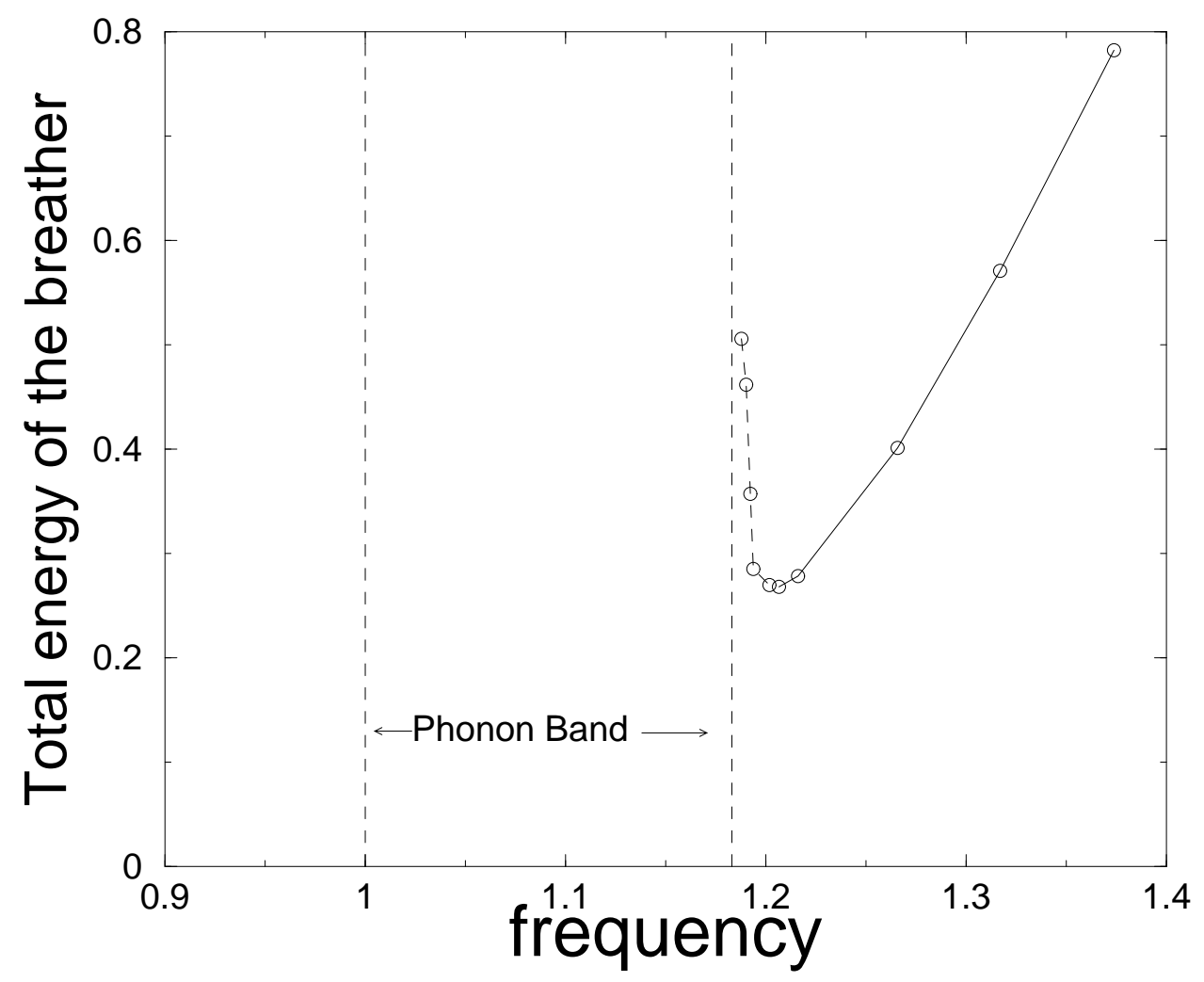

Figure 1: Energy of the breather solution for coupling $k=0.05$ as a function of its frequency (circles). Lines connecting circles are guides to the eye. The dashed vertical lines indicate the boundaries of the phonon band. 

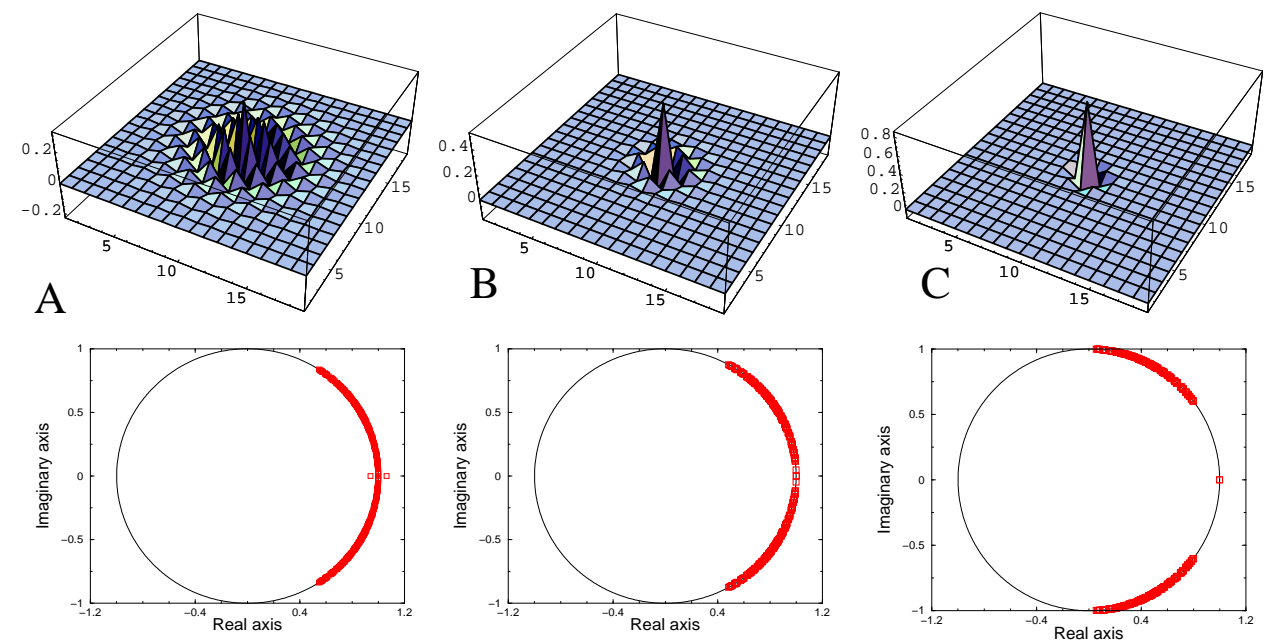

Figure 2: Displacements $u_{l m}(t=0)$ of breather solutions at initial time with zero velocities $\dot{u}_{l m}(t=0)=0$. Below each profile the eigenvalues of the Floquet matrix of linearized perturbations are shown in the complex plane (squares) together with the reference unit circle. A: $\Omega_{b}=1.188$; B: $\Omega_{b}=1.207 ; \mathrm{C}: \Omega_{b}=1.319$.

In terms of energy the threshold value $E_{b, t h}$ provides with a rigorous lower bound - no breathers exist with energies less than $E_{b, t h}$. Consequently we may expect this feature to be detectable in the temperature dependence of equilibrium correlation functions. The corresponding order of magnitude of the average energy per site is expected to be in the region of values $0.05-0.2$. While the upper value is simply close to $E_{b, t h}$, the lower one can be obtained by observing that essentially one central cite and four neighboring sites are important for the dynamics of stable breathers, providing with a lower estimated value of $E_{b, t h} / 5$. At the same time the frequency gap $\omega_{\pi}<\Omega_{b}<\Omega_{b, t h}$ provides with a less rigorous bound. We can only speculate that breathers with frequencies belonging to this gap region are less probable to be excited, because they are linearly unstable. Nevertheless they do exist, and in thermal equilibrium the system may be excited close to these solutions for short times.

\section{Thermal equilibrium}

We thermalize the lattice using as a first method Langevin equations of motion . We add to the right hand side of equations (11) damping terms $-\gamma \dot{u}_{i, j}$ and a Gaussian white noise force $\xi(t)$. The friction is chosen to be $\gamma=0.01$. The Gaussian white noise $\xi$ is characterized by the standard correlation function $\left\langle\xi(t) \xi\left(t^{\prime}\right)\right\rangle=2 \gamma k_{B} T \delta\left(t-t^{\prime}\right)$ where $k_{B}=1$ is the dimen- 


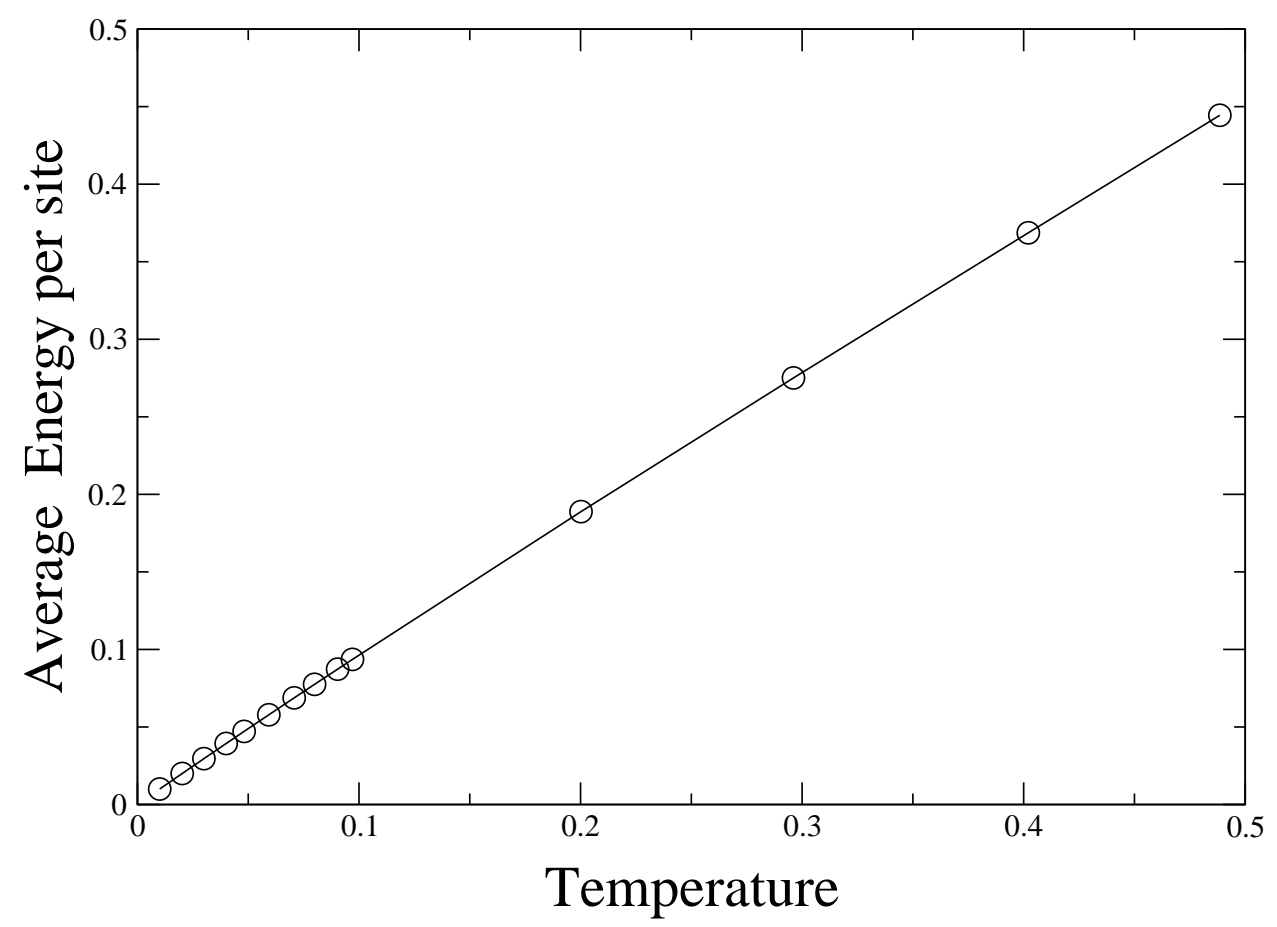

Figure 3: Dependence of the average energy per site $\left\langle h_{l, m}\right\rangle$ on the temperature in the parameter range of interest.

sionless Boltzmann constant and $T$ is the temperature. We simulate the Langevin equations until the averaged kinetic energy per particle is close to the desired value $T / 2$. We then switch off the friction and Gaussian white noise terms, and continue integrating the Hamiltonian equations (1). Time is set to $t=0$ at this moment of switching from Langevin to Hamiltonian evolution. We then measure the time and ensemble averaged kinetic energy and reobtain the corresponding value of $T$, together with computing the time and ensemble averaged energy per site. The temperature values are very close to the corresponding averaged total energies per site $\left\langle h_{l, m}\right\rangle$ as shown in Fig 3

We compute the time dependent displacement-displacement correlation function,

$$
S(t)=\frac{1}{S_{0}} \frac{1}{N^{2}}\left[\frac{1}{t_{f i n}-t_{\text {in }}} \sum_{i=1}^{N} \sum_{j=1}^{N} \int_{t_{\text {in }}}^{t_{f i n}}\left\langle u_{i, j}\left(t+t^{\prime}\right) u_{i, j}\left(t^{\prime}\right)\right\rangle d t^{\prime}\right],
$$

where the brackets denote an ensemble average. In this study the ensemble averaging is obtained by averaging the results for 10 different realizations or runs. The parameters are $t_{i n}=8000$ and $t_{\text {fin }}=8500$. The Fourier 


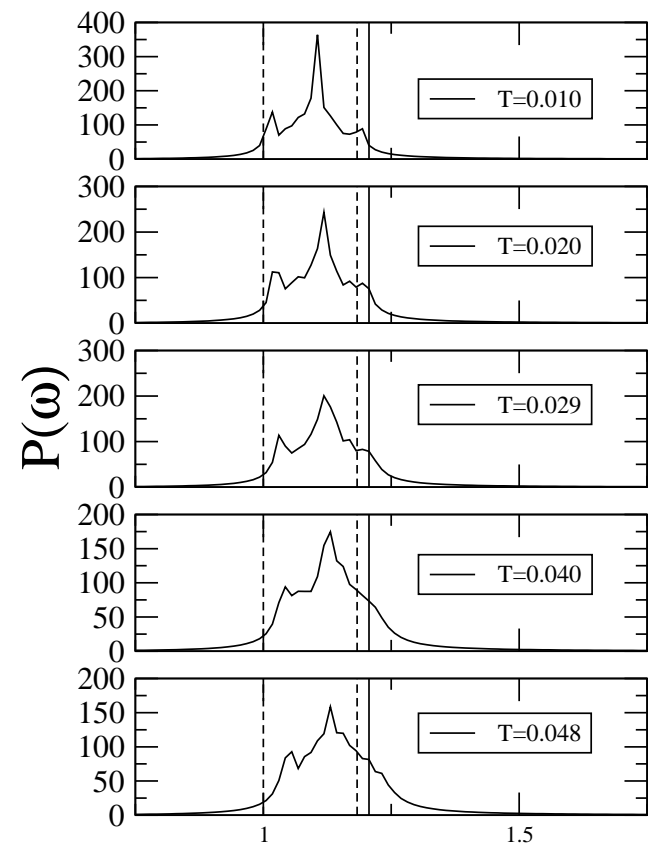

$\omega$

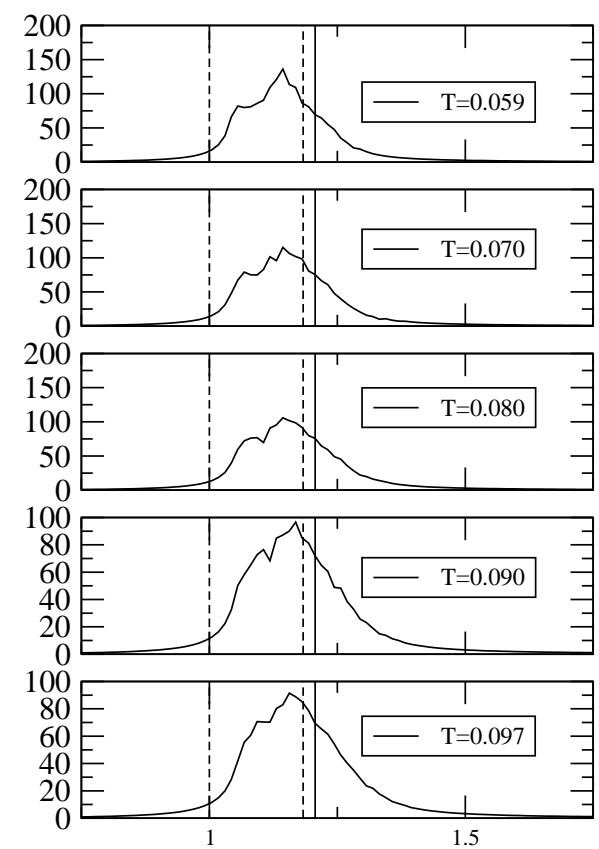

$\omega$

Figure 4: Power spectra $P(\omega)$ as a function of frequency for various values of temperature. The coupling between the sites is $k=0.05$. The values of temperature are indicated in the labels in each subgraph. The two vertical dashed lines mark the band edge values $\omega_{0}$ and $\omega_{\pi}$ of the phonon band while the vertical solid line marks $\Omega_{b, t h}=1.207$.

transform of $S(t)$ is:

$$
S\left(\omega_{m}\right)=\Delta t \sum_{j=0}^{\frac{t_{f i n}}{\Delta t}-1} e^{-\frac{2 \pi i}{t_{f i n}} m \Delta t j} S(\Delta t j),
$$

where $\omega_{m}=\frac{2 \pi m}{t_{f i n}}, m=0,1, \ldots \ldots, \frac{1}{2} \frac{t_{\text {fin }}}{\Delta t}-1$. Finally the power spectrum of the correlation function $S(t)$ is given by

$$
P(\omega)=(\operatorname{Re}(S(\omega)))^{2}+(\operatorname{Im}(S(\omega)))^{2},
$$

where $\operatorname{Re}(S(\omega))$ and $\operatorname{Im}(S(\omega))$ denote the real and imaginary part respectively. The parameter $S_{0}$ is chosen such that $S(t=0)=1$ implying that the frequency integral over $S(\omega)$ is constant.

In Fig 4 we plot the power spectrum $P(\omega)$ for various temperatures. For low temperature values the power spectrum shows that the modes with frequencies inside the phonon band are excited. As the temperature increases the power spectrum is shifted to larger frequencies due to the hard 
anharmonicity. Additionally we observe that for temperatures $T \approx 0.04$ and higher the spectrum exhibits a different behavior in the frequency gap region, viz. shows a decrement near the gap. Let us examine the power

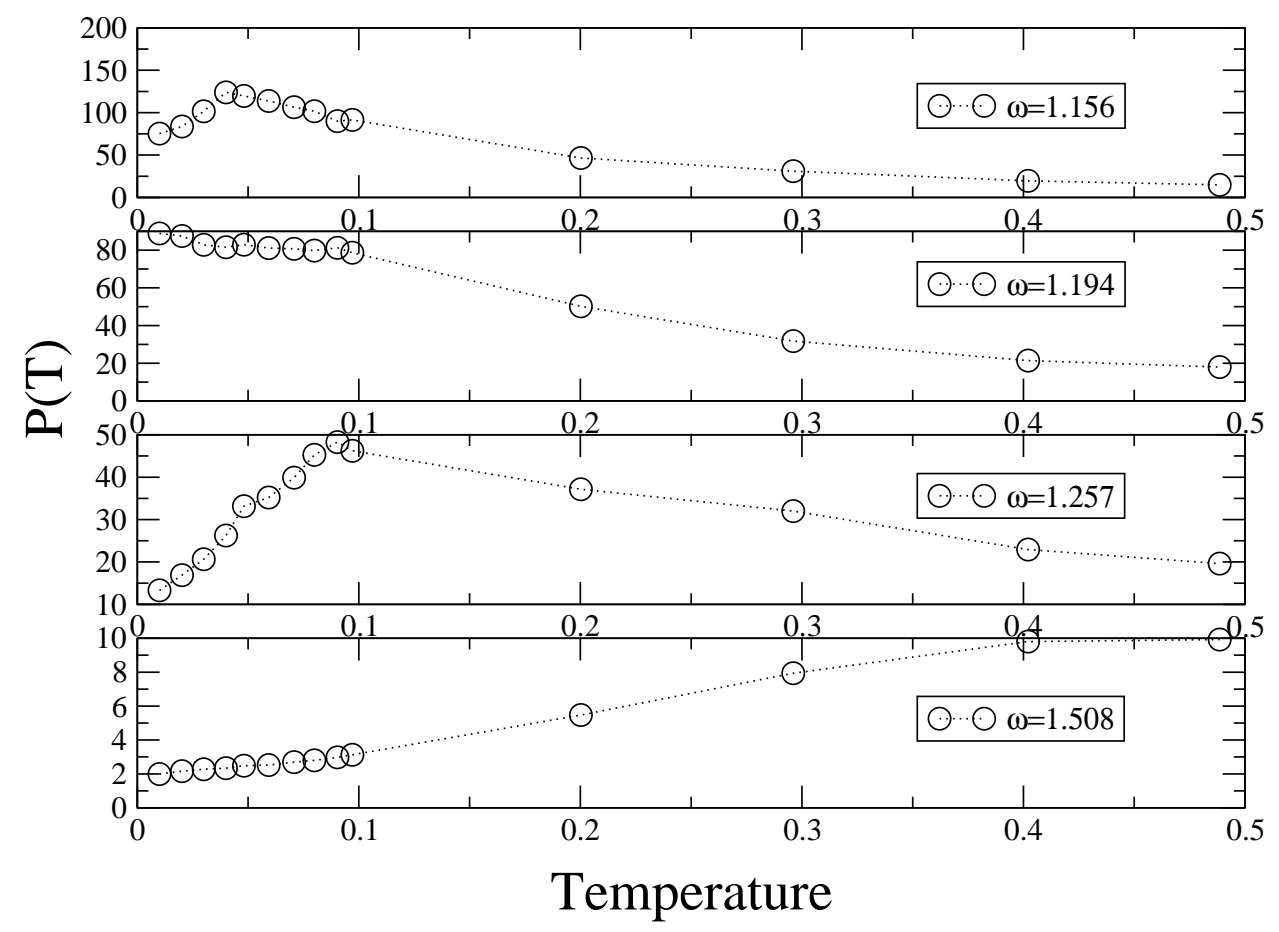

Figure 5: Power spectra $P(T)$ as a function of temperature for 4 different frequencies. From top to the bottom: frequency inside the phonon band, $\omega=1.156$; frequency inside the frequency gap where breathers are unstable $\omega=1.194$; frequency lies outside the two previous regions $\omega=1.257$; largest frequency value $\omega=1.508$.

spectra in detail. In Fig. [5 we plot the value of the power spectrum $P(T)$ as a function of temperature for four specific frequencies. The top panel of Fig. 5 is plotted for a frequency inside the phonon band, the second panel is plotted for a frequency that belongs to the breather frequency gap, while the lower two panels are plotted for frequencies that are located outside the phonon band and the frequency gap region. In the top panel of the Fig. 5 we observe a maximum as the temperature increases due to the shift of the peak of the phonon spectrum with temperature in Fig 4 . This peak is located at $\omega=1.156$ for low temperatures. With increasing temperature the peak passes through the specific frequency that we chose in the top panel of Fig 5. The characteristic temperature value of this peak will depend on the chosen value of the probe frequency inside the phonon band.

In the second panel in Fig [5, where the chosen frequency belongs to the breather frequency gap, a crossover is observed at $T \approx 0.1$. This crossover is 
even more evident in the third panel. In this case the frequency lies outside the gap region but, at the same time, is very close to it. A crossover is observed again at $T \approx 0.1$, a value that has the same order of magnitude as the energy threshold value $E_{b, t h}$ that we found in Section 2. Finally in the bottom panel in Fig [ thace of this threshold is still observed, although the probe frequency is located far from the phonon band and breather frequency gap values, and the power spectrum $P(\omega)$ has only small tails there.

Thus we do observe a characteristic crossover feature in the power spectra, but not as initially expected upon varying the frequency at a fixed temperature. Instead we observe the crossover for a fixed probe frequency by varying the temperature. The absence of a clearly observable frequency gap may be due to the fact that the frequency gap itself is rather narrow, that unstable breathers may still be excited and persist for some time with some probability, and that anharmonic extended waves contribute as well. An interesting question is why the observed crossover upon varying the temperature is showing a maximum or similar cusp for frequencies close to the frequency gap, and an (although bearly visible) opposite behavior at larger probe frequencies (as seen in the bottom panel in Fig [5). A possible answer is that for low temperatures only occasionally breathers are excited, and the shift of frequency contributions to higher values with increasing temperature is as well caused by simple hard anharmonicity effects. However once the temperature reaches the threshold value for breathers, breathers with larger energies are easily excited as well. This will cause a depletion of the power spectra at lower frequencies, at the expense of the increase at higher frequencies, just as observed in Fig 5 . Still the above argumentation shows that it is hard to separate the contribution of anharmonic phonons from that of breathers. We clearly are in need of a technique which does this separation. The next chapter will provide with a solution to this problem.

\section{How to measure breathers in thermal equilib- rium}

As already discussed in the introduction, DBs act as strong point-like scatterers of plane waves [15. In one-dimensional lattices this circumstance makes it hard to let the delocalized radiative part of excitations out of the system [19. However in two-dimensional (and even more efficiently in threedimensional) systems point-like scatterers will not hinder plane waves from moving around such an obstacle. Consequently we may attach dissipative boundaries to our lattice, and expect the radiation to disappear from the system during a reasonable short time, leaving the immobile localized excita-

tions behind. Such cooling techniques have been used for studying breather relaxations [16, 18, 19. Here we are only interested in letting the radiation out and being left with DBs. 
We study an ensemble of 10 different realizations that correspond to the same initial energy per site $E_{0}$. We thermalize the system in another way here. We start the system with a given initial energy $E_{0}$, i.e. we integrate the system for 1000 time units using initially random displacements for the particles. After that dissipative boundaries are switched on for a given transient of time. This is done here by adding a friction term to the boundary sites of a $20 \times 20$ lattice with friction constant 0.1 for some cooling time $T_{\text {cool }}$. While much more sophisticated ways of reflectionless dissipative boundaries can be implemented [21], this simple method suffices for the results presented below.

The effect of the dissipative boundary is shown in Figs 6 . 7 where the energy per site remaining in the lattice is plotted as a function of time, both in normal and in logarithmic units. Note that the energy values are normalized to their corresponding number at time $t=0$ at which the dissipative boundary is switched on.

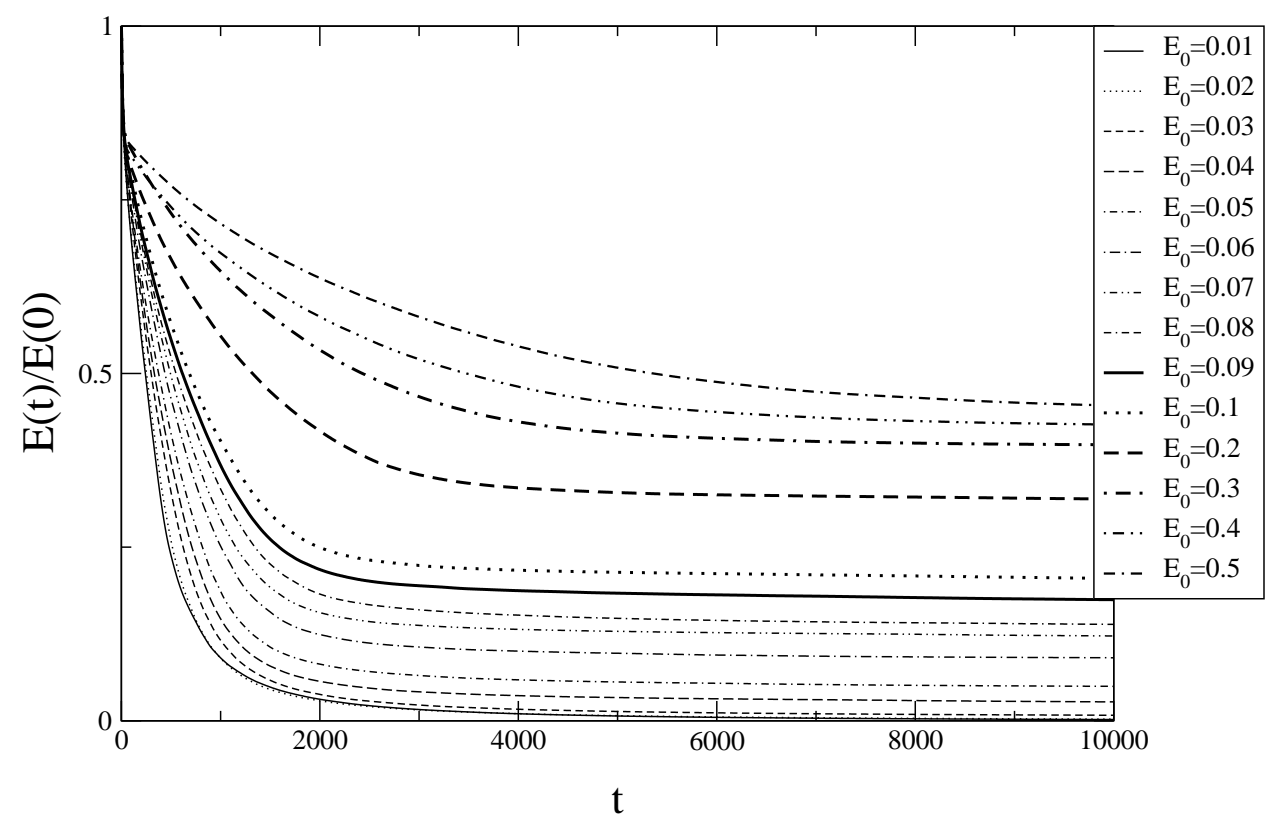

Figure 6: The time dependence of the normalized lattice energy per site in the presence of a dissipative boundary for different values of the initial energy per site as indicated in the figures.

We clearly observe that after some transient behavior the lattice energy density changes rather slowly, indicating the expected outcome - delocalized excitations left the system, leaving the localized ones behind. Both Fig 6 and especially Fig[7] show that the characteristic waiting time increases with 


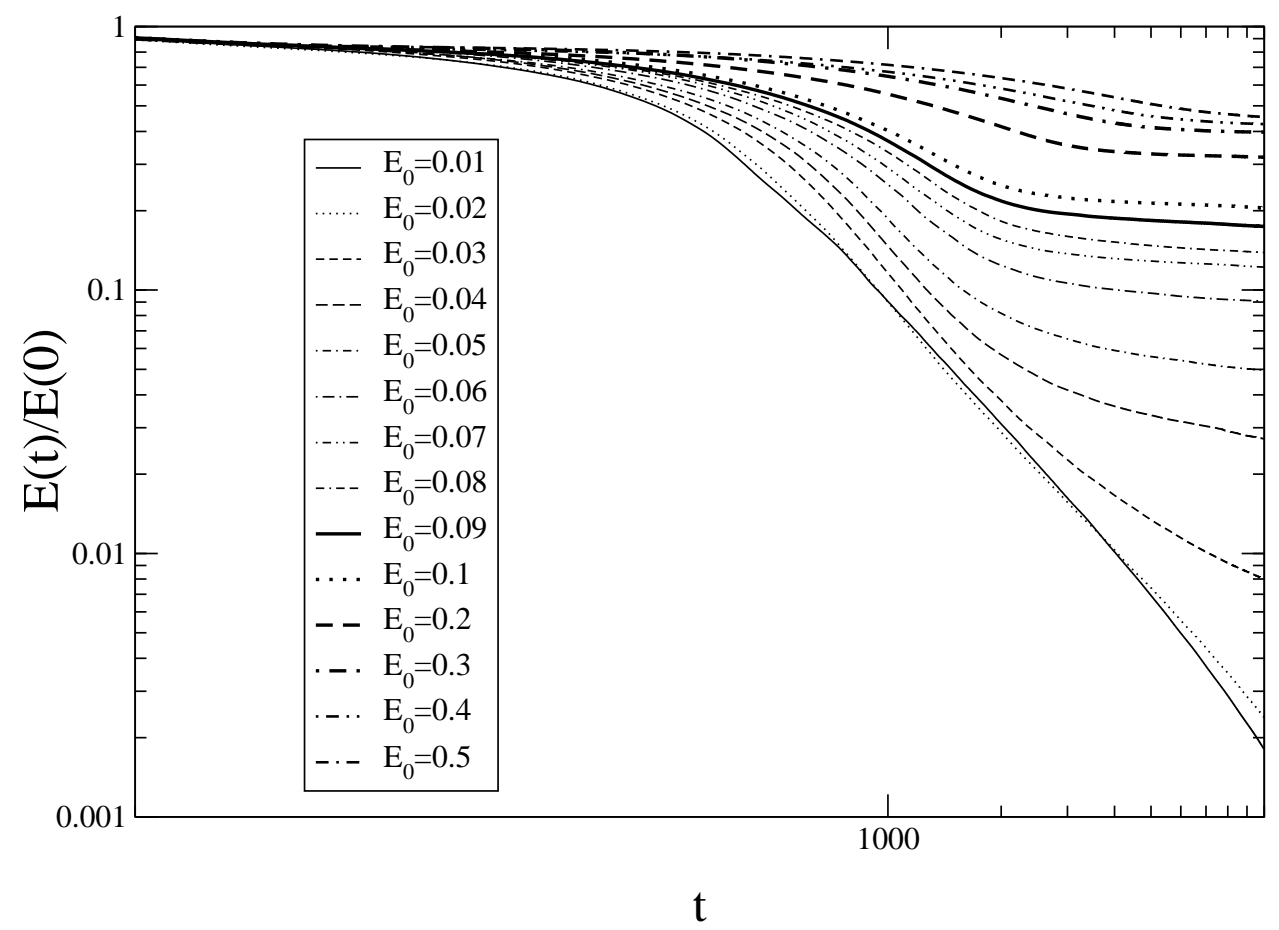

Figure 7: Same as in Fig [ but in logarithmic units.

increasing initial energy density from roughly $T_{\text {cool }}=2000$ up to $T_{\text {cool }}=$ 10000. This can be observed from the shift of the inflection points in Fig 7 In the following we will use a cooling time $T_{\text {cool }}=10^{4}$, but we will also compare with shorter cooling times.

In Fig. 8 we plot the total energy that remains in the lattice (excluding the sites that belong to the boundaries) per site and per number of realizations $E$ as a function of initial energy $E_{0}$. Note the log scale for the y-axis. For clarity the inset is for the same data but with linear axis scaling. We observe a crossover for $E_{0} \sim 0.05$. We fit the curve of $E\left(E_{0}\right)$ first using

$$
E=A \tilde{E}_{b, t h} e^{-\frac{\tilde{E}_{b, t h}}{E_{0}}}
$$

with parameters A and $\tilde{E}_{b, t h}$. This holds assuming that only breathers with $\tilde{E}_{b, t h}$ will be excited. Then the probability to form a DB is $e^{-\frac{\tilde{E}_{b, t h}}{E_{0}}}$ while its contribution to an energy distribution will be $\tilde{E}_{b, t h} e^{-\frac{\tilde{E}_{b, t h}}{E_{0}}}$. Depending on the energy range we use for fitting we obtain $\tilde{E}_{b, t h}=0.17\left(E_{0}<0.06\right)$ or $\tilde{E}_{b, t h}=0.233\left(E_{0}<0.1\right)$. We were not able to fit data at larger energies. The obtained values $0.17-0.233$ are in good agreement with the expected value of the DB energy threshold 0.27. The dashed line in Fig 8 is the corresponding fit with parameters $A=0.175$ and $\tilde{E}_{b, t h}=0.198$. 


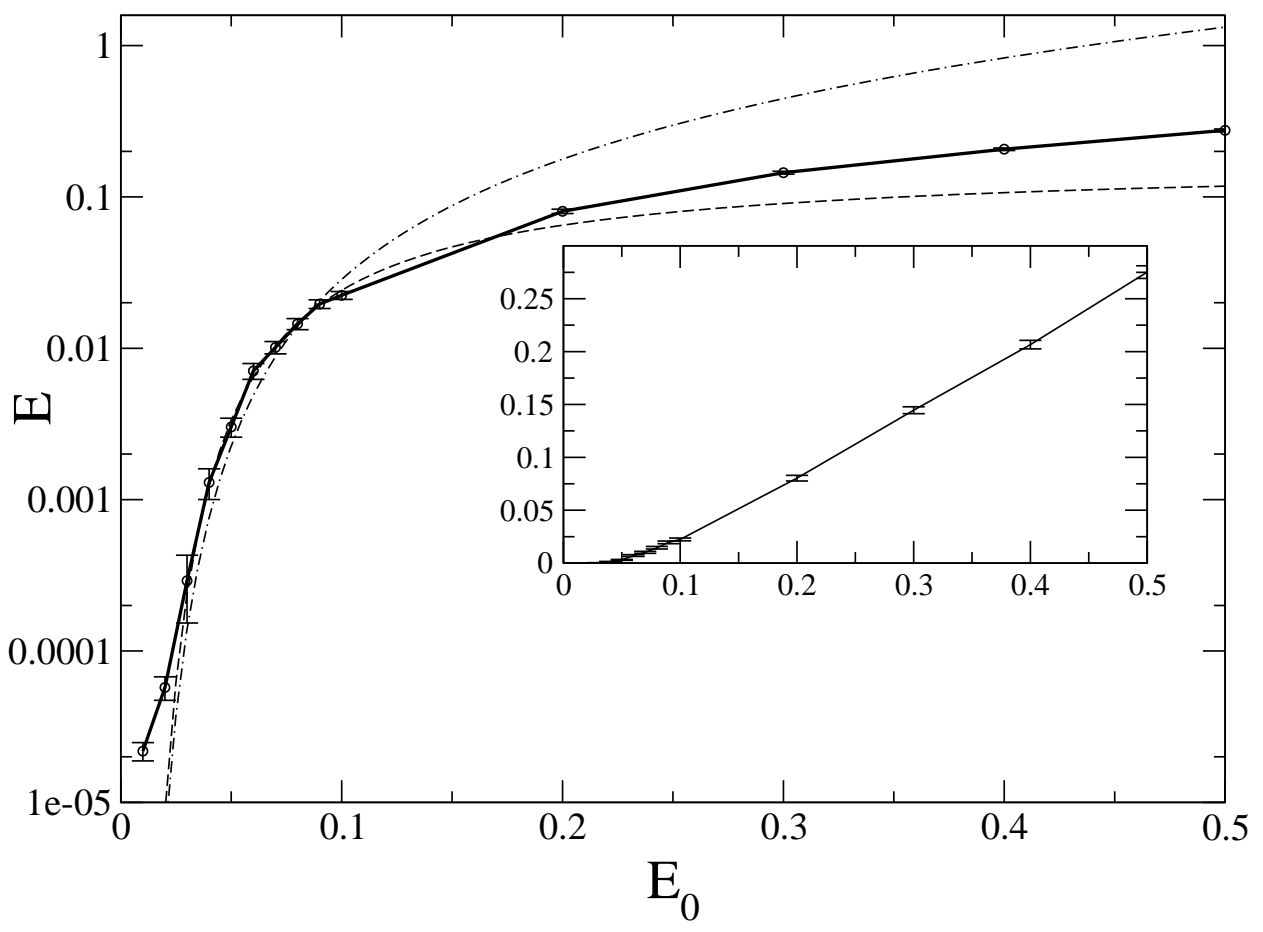

Figure 8: Graph of the average energy per site and per number of realizations $E$ as a function of the initial energy $E_{0}$ after cooling. The error bars are the standard deviations of the mean for the statistical ensemble. Note the logarithmic scale of the y-axis. Solid line -guide to the eye. Dashed line fit using (7). Dashed-dotted line - fit using (8) (for parameters see text). Inset: Same but with linear y-axis scaling to observe the crossover around $E_{0}=0.05$.

In a refined fitting we allow also for breathers with larger energies. Then the energy per site contribution to $E$ will be given by

$$
A \int_{\tilde{E}_{b, t h}}^{\infty} E_{b} \mathrm{e}^{-\frac{E_{b}}{E_{0}}} \rho\left(E_{b}\right) d E_{b},
$$

where $\rho\left(E_{b}\right)$ is the density of DB states. Assuming $\rho\left(E_{b}\right)=1$ (its actual constant value can be always absorbed in $A)$, (8) yields $A\left(E_{0} E_{b, t h}+E_{0}^{2}\right) \mathrm{e}^{-\frac{\tilde{E}_{b, t h}}{E_{0}}}$ and the subsequent fitting procedure results similar results for $\tilde{E}_{b, t h}$ as obtained with (7). The dashed-dotted line in Fig 8 is the corresponding fit with parameters $A=5.53$ and $\tilde{E}_{b, t h}=0.163$. While the low energy region of the numerical data is well reproduced, the high energy data are underestimated with (7) and overestimated with (8). This implies that the density $\rho\left(E_{b}\right)$ is not constant, but decaying with increasing energy $E_{b}$.

But we can obtain even more relevant data. After the cooling process we measure all values $h_{l, m}$ of the energy density. In Figs. 9 and 10 we present 
their distribution $W(E)$ additionally averaged over all the realizations after cooling. The initial energy per site that is used is indicated in the labels in each subfigure. Note that the data are obtained by coarsegraining the energy axis with a grid size of 0.05 . The data obtained within the first box $0<E<0.05$ are omitted from the plots (except for the two left upper panels at the two lowest energies $E_{0}$ ).

The DB energy threshold is visible in all the subgraphs except the first two subgraphs where the initial energy $E_{0}$ is very low and DBs cannot be formed. We also clearly see that already for energies $E_{0}=0.04$ a whole distribution of breather energies is obtained, with maximum breather energies being twice larger compared to the minimum DB energy. Note that the observed energy gaps are very close to the value 0.17 . This corresponds exactly to the expected energy of the central site for the minimum energy breather being equal to 0.167 (see Fig $2 \mathrm{~B}$ ). The nearest neighbours of such a breather carry approximately ten percent of the full DB energy each, i.e. 0.027. Their contributions are thus within the mentioned first box and not present in the plotted data.
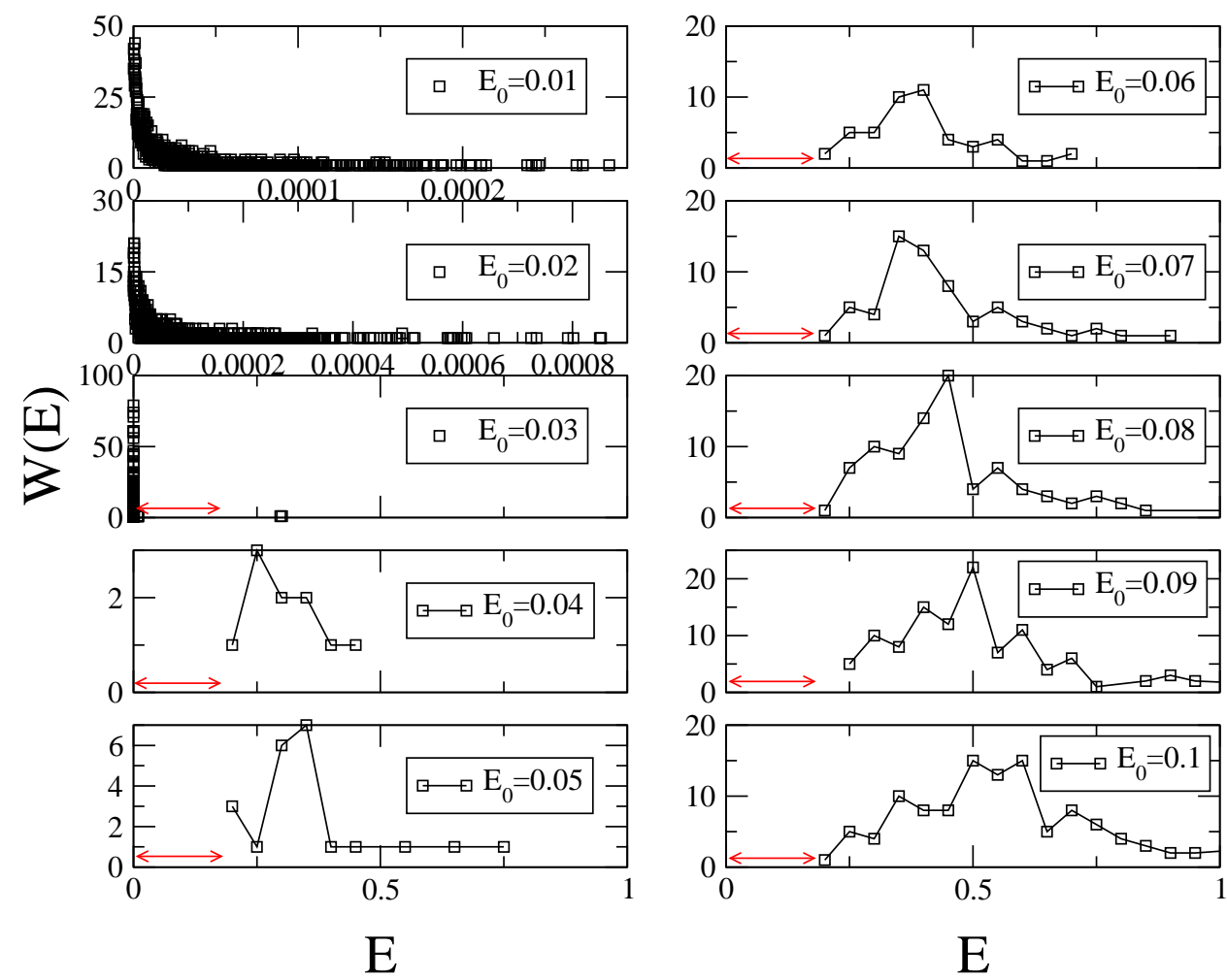

Figure 9: Graph of the energy distribution $W(E)$. See text for details. The arrows have length 0.17 and show the energy region below which the distribution is expected to vanish due to the DB energy threshold. 

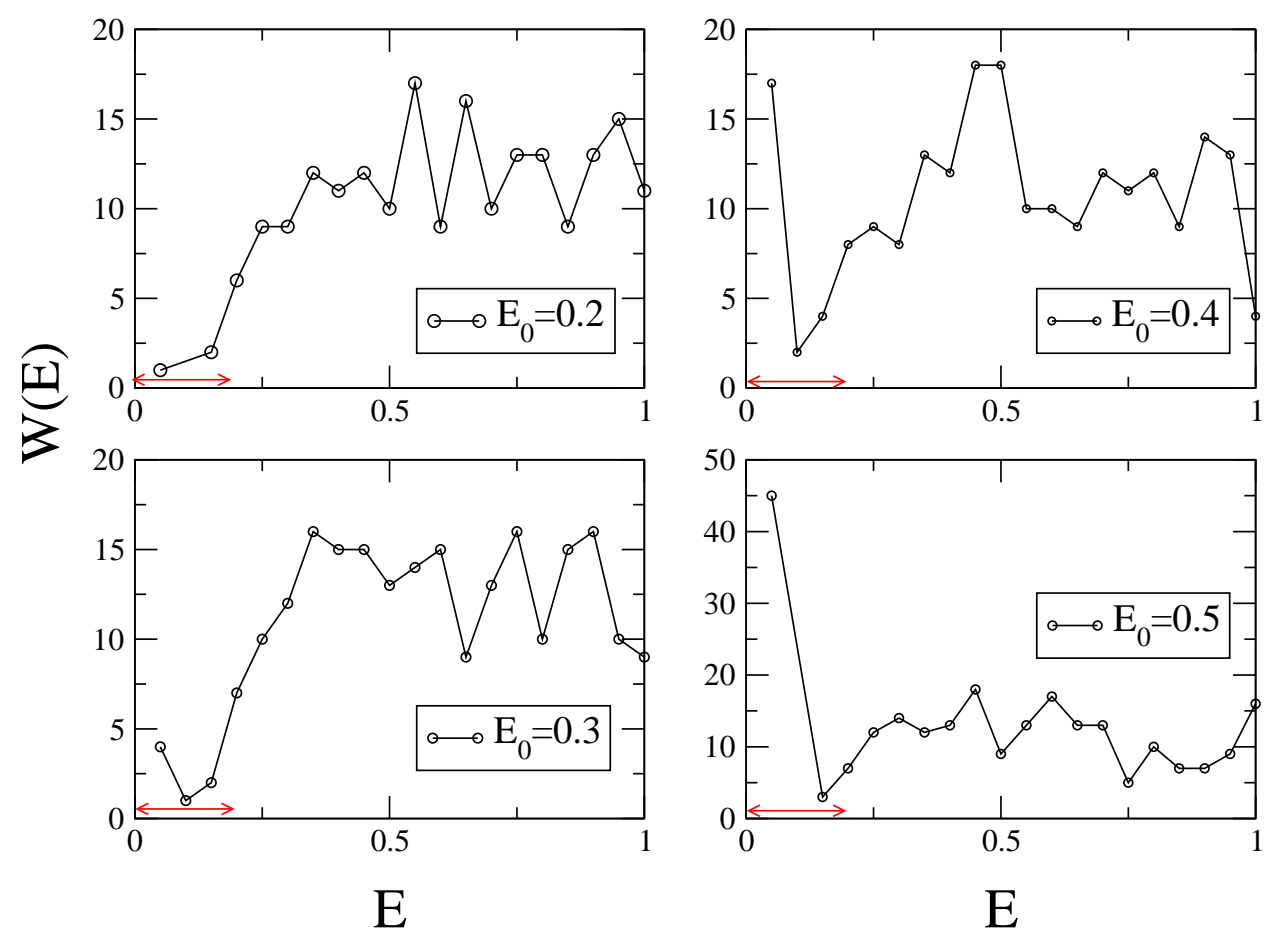

Figure 10: Graph of the energy distribution $W(E)$ as in Fig. 9 but for higher initial energy $E_{0}$. 

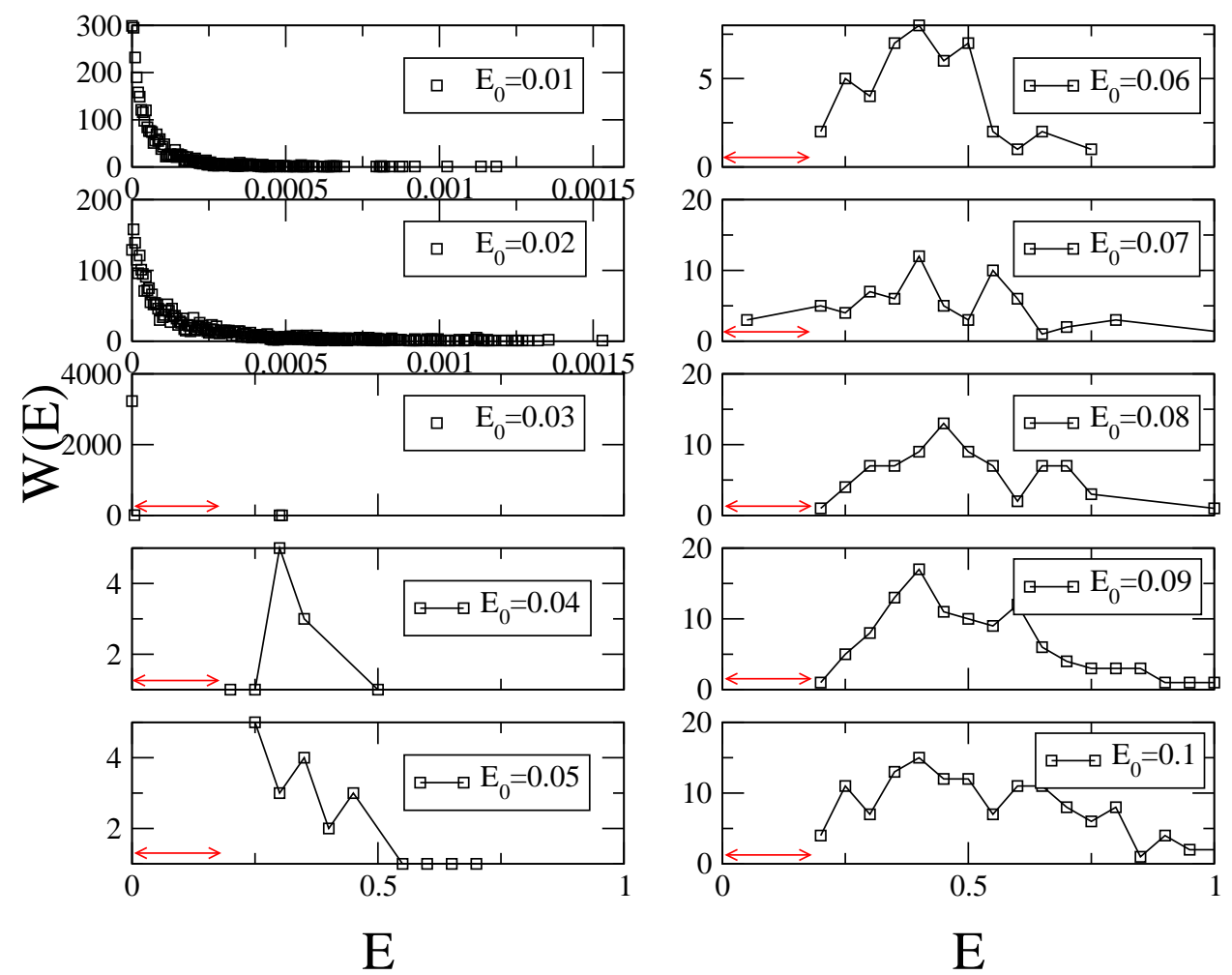

Figure 11: Graph of the energy distribution $W(E)$ as in Fig. 9 but for $T_{\text {cool }}=5000$.

In order to test the sensitivity of these data on the cooling time $T_{\text {cool }}$ we present in Figs 1112 similar results for $T_{\text {cool }}=5000$ which is twice smaller. While the overall statistics needs to be improved in both cases, we find semiquantitative agreement. This indicates that during the rather long cooling times the statistical properties of the localized excitations remaining in the lattice are not significantly changing. The observed increase of $W(E)$ for small $E$ and large temperatures may be due to the fact that at these large temperatures the cooling time $T_{\text {cool }}$ was too short, leaving delocalized excitations inside the system. We will provide with further evidence for the correctness of this conclusion.

The above analysis suggested that to some accuracy the excitations in the lattice at thermal equilibrium can be considered as a sum of localized and delocalized excitations. The power spectra in Fig 4 represent thus a sum of the power spectra of both types of excitations. We use now the cooling process, which leaves us with the localized excitations only. We wait for $T_{\text {cool }}=8000$ and after that compute the power spectrum of the remaining localized excitations in the system. The results are shown in Figs 13 14. The comparison of Figs 1314 with Fig 4 shows, that for energies $E_{0}<0.03$ 

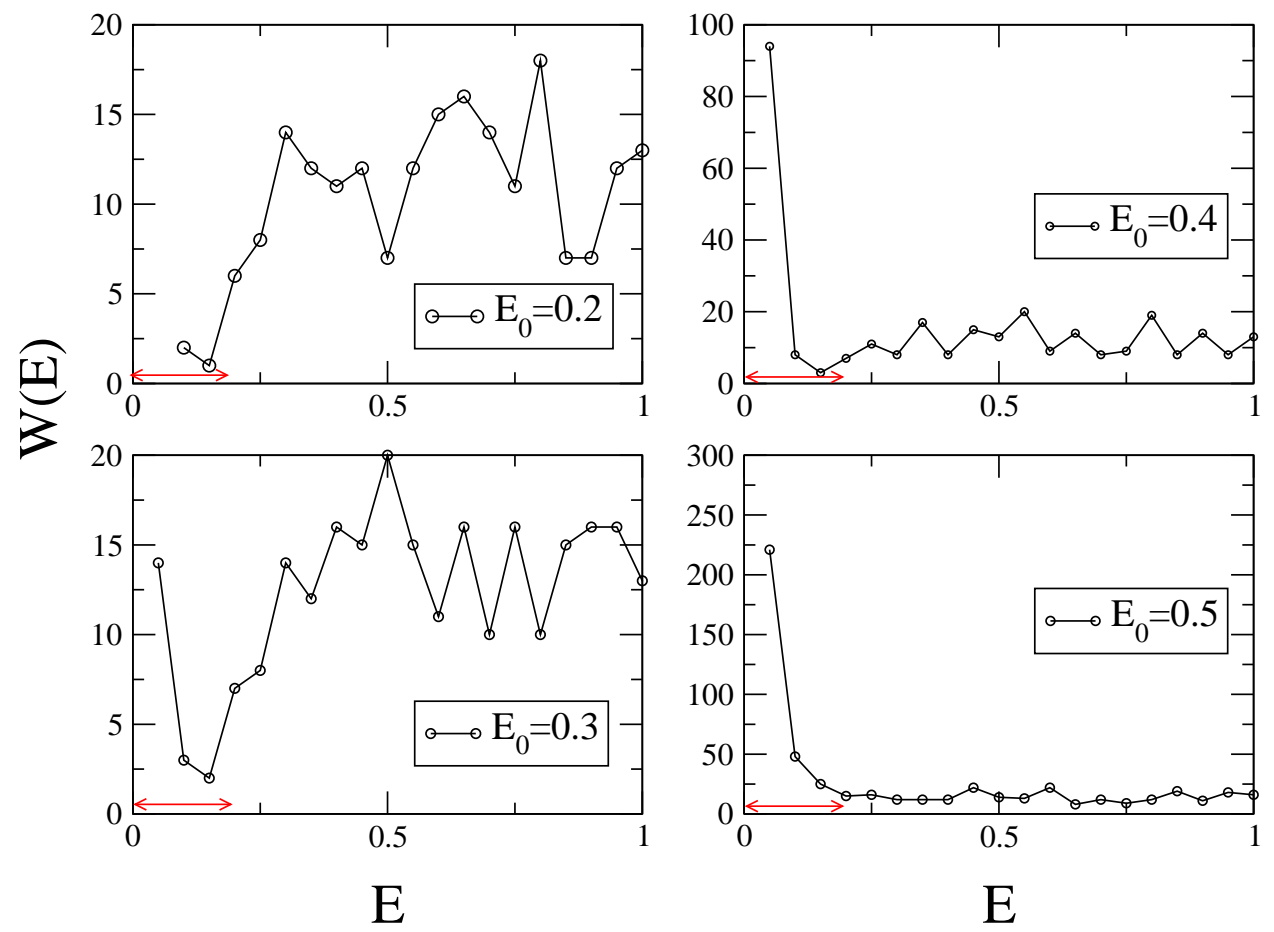

Figure 12: Graph of the energy distribution $W(E)$ as in Fig 10 but for $T_{\text {cool }}=5000$. 


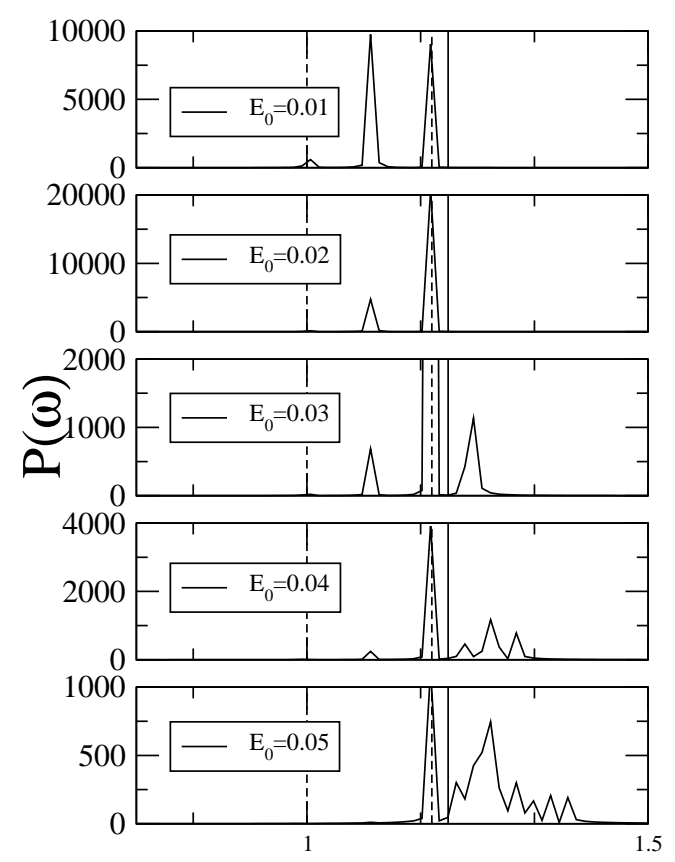

$\omega$
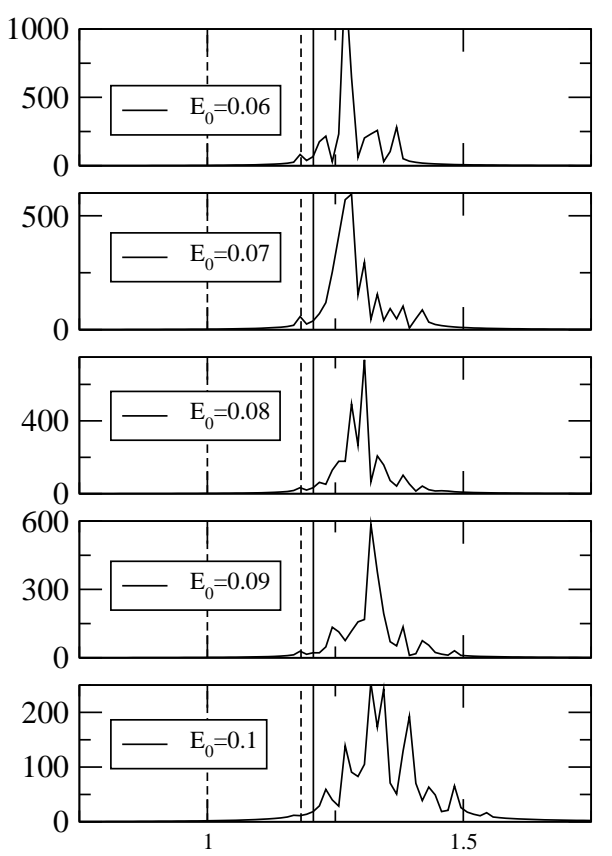

$\omega$

Figure 13: Same as in Fig 4 but after $T_{\text {cool }}=8000$.
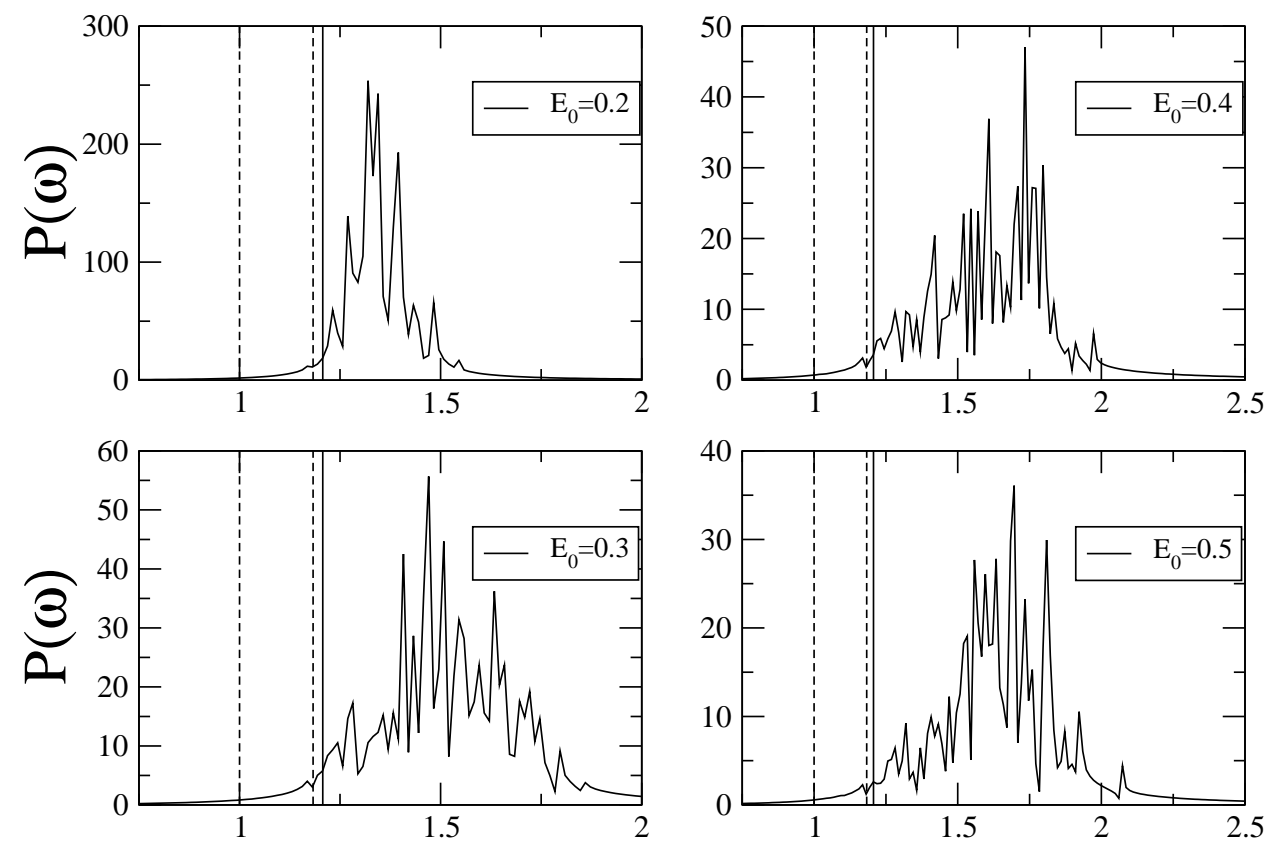

$\omega$

$\omega$

Figure 14: Same as in Fig 4 but after $T_{\text {cool }}=8000$. 
i) the dynamics is essentially governed by delocalized excitations; ii) after the cooling period only delocalized states with almost zero group velocities remain in the system (these correspond to the band edge frequencies and to the peak inside the band). For larger energies $E_{0}$ the power spectra after cooling show the existence of localized excitations with frequencies outside the band $\omega_{\vec{q}}^{2}$. Moerover, we observe very clearly that the frequency gap $\omega_{\pi}<\Omega_{b}<\Omega_{b, t h}$ is depleted here, indicating that unstable DBs decay during the cooling time and radiate their energy into the dissipative boundary, even if they were present to some extent in thermal equilibrium. Thus the presented way of separating localized from delocalized excitations in thermal equilibrium allows also to quantitatively determine the contribution of DBs to correlation functions. Finally we observe that at the largest temperatures considered, some nonzero statistical weight is observed in the frequency region of the phonon band and even below it. This confirms our previous expectation, that at these large temperatures the cooling times are probably not long enough to let the extended excitations out of the system. A possible reason may be that at these large temperatures many DBs are coexisting in the system. Extended waves will spend more time to diffuse around these scattering centers. DBs could even form temporary percolation networks which would hinder the escape of waves even more efficiently.

\section{Conclusions}

Our results show that discrete breathers (DBs) leave clear and detectable fingerprints in the thermal equilibrium properties of nonlinear lattices. Using the case of a two-dimensional lattice, we demonstrated the persistence of an energy threshold for the existence of DBs in time-dependent correlation functions by observing weak crossover features. Moreover, we used the technique of boundary cooling to separate the localized excitation part at thermal equilibrium from the delocalized ones. This allows us to study statistical properties of DB excitations in thermal equilibrium, e.g. their contribution to the abovementioned correlation functions. This simple step allowed us to unambiguously confirm the presence of the energy threshold for DBs in thermal equilibrium. We furthermore confirm that the frequency gap (which corresponds to the excitation of unstable DBs) is depleted after the separation procedure. Thus we conclude that unstable DBs are not contributing to dynamical correlations at thermal equilibrium. By comparing the spectra of the system at thermal equilibrium with the spectra of the DB part only, we can provide with reliable statistical weights of both the delocalized and localized excitation parts of the system at thermal equilibrium at one and the same frequency. It seems to be very difficult to provide with a similar cooling and separation procedure in any realistic experimental setup, despite the fact that DBs have been observed in a variety of different 
systems. That makes computational studies of DB properties at thermal equilibrium a unique way of gaining further understanding of the relaxation and excitation properties of complex lattices.

With this simple technique, which certainly needs more refinement, we are now able to reliably compute various statistical contributions of DBs, including also correlation effects between DBs and delocalized excitations. The door is thus open for starting serious analytical work on DBs in thermal equilibrium, since the presented new numerical tools will allow for a much more refined testing of various theories as compared to simulations of thermal equilibrium only.

Acknowledgements

We thank V. Fleurov, G. Kalosakas, R. Livi and G. Tsironis for useful discussions.

\section{References}

[1] A. J. Sievers and J. B. Page, in: Dynamical properties of solids VII phonon physics the cutting edge, Elsevier, Amsterdam (1995); S. Aubry, Physica D 103, 201 (1997); S. Flach, C.R. Willis, Phys. Rep. 295, 181 (1998); Energy Localisation and Transfer, Eds. T. Dauxois, A. LitvakHinenzon, R. MacKay and A. Spanoudaki, World Scientific (2004); D. K. Campbell, S. Flach and Yu. S. Kivshar, Physics Today, p.43, January 2004

[2] P. Binder, D. Abraimov, A. V. Ustinov, S. Flach, and Y. Zolotaryuk, Phys. Rev. Lett. 84, 745 (2000); E. Trias, J. J. Mazo, and T. P. Orlando, Phys. Rev. Lett. 84, 741 (2000); A. Ustinov. Chaos 13, 716 (2003).

[3] H. S. Eisenberg et al., Phys. Rev. Lett. 81, 3383 (1998); R. Morandotti et al., Phys. Rev. Lett. 83, 2726 (1999); 83, 4756 (1999); J. W. Fleischer, M. Segev, N. K. Efremidis, and D. N. Christodoulides, Nature 422, 147 (2003); D.Cheskis et.al., Phys. Rev. Lett. 91, 223901 (2003).

[4] B. Swanson et al., Phys. Rev. Lett. 82, 3288 (1999); K. Kladko, J. Malek and A. R. Bishop, J. Phys.: Condens. Matter 11, L415 (1999).

[5] U.T. Schwarz, L.Q. English, and A.J. Sievers, Phys. Rev. Lett. 83, 223 (1999).

[6] M. Sato, B. E. Hubbard, A. J. Sievers, B. Ilic, D. A. Czaplewski, and H. G. Craighead, Phys. Rev. Lett. 90, 044102 (2003); M. Sato, B. E. Hubbard, A. J. Sievers et al., Europhys. Lett. 66, 318 (2004). 
[7] B. Eiermann, Th. Anker, M. Albiez, M. Taglieber, P. Treutlein, K.-P. Marzlin and M. K. Oberthaler, Phys. Rev. Lett. 92, 230401 (2004).

[8] M. Machida and T. Koyama, Phys. Rev. B 70, 024523 (2004).

[9] I. Kourakis and P. K. Shukla, Phys. Plasmas 11, 1384 (2004).

[10] V. M. Burlakov, S. Kisilev and V. N. Pyrkov, Solid State Comm. 74, 327 (1990); V. M. Burlakov, S. Kisilev and V. N. Pyrkov, Phys. Rev. B 42, 4921 (1990); T. Dauxois, M. Peyrard and A. R. Bishop, Phys. Rev. E 47, 684 (1993); S. Flach and G. Mutschke, Phys. Rev. E 49, 5018 (1994); M. Peyrard, Physica D 119, 184 (1998); M. Peyrard and J. Farago, Physica A 288, 199 (2000).

[11] K. O. Rassmussen, T. Cretegny, P. G. Kevrekidis and N. GronbechJensen, Phys. Rev. Lett. 84, 3740 (2000); B. Rumpf and A. C. Newell, Phys. Rev. Lett. 87, 054102 (2001); B. Rumpf and A. C. Newell, Physica D 184, 162 (2003); B. Rumpf, Phys. Rev. E 69, 016618 (2004).

[12] G. Kalosakas, K. O. Rasmussen and A. R. Bishop, J. Chem. Phys. 118, 3731 (2003).

[13] S. Flach, K. Kladko and R. S. MacKay, Phys. Rev. Lett. 78, 1207 (1997).

[14] M. Eleftheriou, S. Flach, G. P. Tsironis, Physica D 186, 20 (2003).

[15] T. Cretegny, S. Aubry and S. Flach, Physica D 119, 73 (1998); S. Flach, A. E. Miroshnichenko and M. V. Fistul, CHAOS 13, 596 (2003).

[16] G. P. Tsironis and S. Aubry, Phys. Rev. Lett. 77, 5225 (1996); F. Piazza, S. Lepri and R. Livi, J. Phys. A 34, 9803 (2001); R. Reigada, A. Sarmiento and K. Lindenberg, Phys. Rev. E 64, 066608 (2001); R. Reigada, A. Sarmiento and K. Lindenberg, Phys. Rev. E 66, 046607 (2002).

[17] F. Piazza, S. Lepri and R. Livi, Chaos 13, 637 (2003).

[18] A.Bikaki, N. K. Voulgarakis, S. Aubry and G. P. Tsironis, Phys. Rev. E 59, 1234 (1999).

[19] M. V. Ivanchenko, O. I. Kanakov, V. D. Shalfeev and S. Flach, Physica $\mathrm{D}$, in print.

[20] G. Kalosakas, S. Aubry and G. P. Tsironis, Phys. Rev. B 58, 3094 (1998).

[21] S. Flach, K. Kladko and C. R. Willis, Phys. Rev. E 50, 2293 (1994). 